Gynaecologia 1958;146:469-470

\title{
Register nominum
}

Adler, U., und Nick, J., 283 (S)

-, v. Nick, J.

Anderes, W., 343

Andreas, H., 116

Backhausz, R., und Neubauer, G., 243

Berger, M., v. Linder, A.

Beric, B., v. Böttger, $\mathrm{H}$.

Bóna, E., 47

Böttger, H., und Beric, B., 361

Bromberg, Y. M., and Erdreich, M. B.,

222 Brume, T. L. A. de, 152 Champod, Y., v. Rossel, G. Cottier, l·L, v. Müller, C. Curchod, A., v. Rossel, G. Delinotte, P., 273 (S)

Erdreich, M. B., v. Bromberg, Y. M.

Fischer, W., 410 (B)

Friedberg, V., 431

Grüninger, B., v. Koller, T.

Hauser, G. A., Stingelin, A., Keller, M.,

und Wenner, R., 323 (S) -, v. Klinger, H. P. Held, E., 273 (S) Herrmann, U., 318 (S) Hochstaedt, B., und Langer, G., 372 Hofstätter, R., 256 Homma, H., 193 Huber, A., Ill Huggenberg, H. R., und Kesselring, F.,

312 (S) ,fakobovits, A., 440

470 Register

Joel, Ch. A., 53

-, und Lancet, M., 425

Kaiser, R., 342 (B)

Käser, 0., und Kaufmann, P., 279 (S)

Kaufmann, P., v. Käser, O.

Keller, M., v. Hauser, G. A.

Kern, E., v. Wiemers, K.

Kesselring, F., v. Huggenberg, H. R.

Keßler, R., v. Wagner, $\mathrm{H}$.

Klinger, H. P., Ludwig, K. S.,

Schwarzacher, H. G., und Hauser,

G. A., 328 (S) Koller, T., und Grüninger, B., 163 Koutský, J., 399 Kulitzy, G., 231 Lancet, M., v. Joel, Ch. A. Langer, G., v. Hochstaedt, B. Lederrey, P., Voegeli, l·L, et

Watteville, H. de, 301 (S) Leinzinger, E., 103 Lewin, H., 42

Linder, A., und Berger, M., 411 Louros, N. C, 270 (B) Ludwig, K. S., v. Klinger, H. P. Martinet, R., 289 (S) Molnar, R., 157 Müller, C, und Cottier, H., 93 Neubauer, G., v. Backhausz, R. nommum 
Nick, J., und Adler, U., 296 (S)

-, v. Adler, U.

Rawyler, V., v. Stamm, O.

Reist, A., 342 (B)

Rieben, G., 309 (S)

Rossel, G., et Champod, Y., 301 (S)

-, et Curchod, A., 307 (S)

Rütte, B. von, 291 (S)

Salvatierra, V., et Sureda, G., 31

Scharplatz, A., 335 (S)

Schultheiß, H. R., 382

Schwarzacher, H. G., v. Klinger, H. P.

Sillo, G., 60

Staemmler, H.-J., 1

Stamm, H., 266, 406

Stamm, 0., et Rawyler, V., 317 (S)

Stingelin, A., v. Hauser, G. A.

Storz, B., 134

Sureda, G., v. Salvatierra, V.

Vara, P., 72, 174

Verschoof, K. J. H., 453

Voegeli, H., v. Lederrey, P.

Wagner, H., und Keßler, R., 459

Watteville, H. de, v. Lederrey, P.

Wenner, R., v. Hauser, G. A.

Wiemers, K., und Kern, E., 410 (B)

Wiqvist, N., 65

Zürcher, P., 340 (S) 